\title{
Gamma-Induced Modification on Optical Band Gap of CR-39 SSNTD
}

\author{
M. F. Zaki* \\ Experimental Nuclear Physics Department, \\ Nuclear Research Center, Atomic Energy Authority, \\ P. O. 13759 Abu Zaabal, Cairo, Egypt \\ (Received on 13 May, 2008)
}

\begin{abstract}
Effect of gamma irradiation on optical absorption of nuclear track detectors like CR-39 was studied in different absorbed doses using ultraviolet-visible (UV-VIS) spectroscopy. The existence of the peaks, their shifting and broadening as a result of gamma irradiation has been discussed. The width of the tail of localized states in the band gap $\left(E_{u}\right)$ was evaluated using the Urbach edge method. Finally the indirect and direct band gap in pristine and gamma irradiated CR-39 have been determined. The values of indirectly band gap have been found to be lower than the corresponding values of direct band gap. A decrease in the optical energy gap with increasing the gamma absorbed dose can be discussed on the basis of gamma-irradiation-induced defects in the CR-39. The correlation between optical band gap and the number of carbon atoms in a cluster with modified Tauc's equation has been discussed in case of CR-39.
\end{abstract}

Keywords: Optical absorption; CR-39; UV-VIS spectra; Optical band gap

\section{INTRODUCTION}

The importance of polymers has increased very rapidly during the last few decades because of their low cost, easy processability, low weight, high quality surfaces and easy fabrication of thick and thin samples, etc. One of the most important polymers is CR-39, which is used in different applications. These applications include up-to-date studies in nuclear and fission physics; alpha, neutron, and charged-particle radiography; cosmic ray studies and astrophysics; porosity and microfilters; alpha- and neutron-dosimetry; uranium prospection and radon-emanation measurements; radiological protection and monitoring; radiobiology and nuclear medicine measurements made on meteorites, lunar samples, in stratospheric balloons and from space vehicles, as well as search for superheavy elements. When a polymeric nuclear track detector, CR-39, is exposed to high intensity gamma radiations, induces modifications in their structural, physical and chemical properties $^{(1)}$. The changes in these properties after exposure to high energy radiation have been evaluated by many techniques. The effect of gamma radiation on the physical and chemical properties of polymers has been reported ${ }^{(2,3)}$. At the same time knowledge of this effect may offer the prospects for measuring gamma dose.

It is already an established fact that interaction of radiation with polymers leads to chain scission, chain aggregation, formation of double bonds and molecular emission. As a consequence of this, the physico-chemical properties like optical, electrical, mechanical, chemical and track properties of the polymer are modified ${ }^{(4-12)}$. The effectiveness of these changes produced depends upon the structure of the polymer and the experimental conditions of irradiation like energy and fluence. The study of these changes may enhance their applications in different fields, e.g. for the evolution of high radiation $\operatorname{doses}^{(13,14)}$.

*Electronic address: moha1016@yahoo.com
Clusters are small nano-size particles containing fewer than $10^{4}$ atoms or molecules. Such clusters are known to be formed along latent tracks of energetic ions in polymers ${ }^{(15-17)}$. Overlapping tracks formed by irradiation with low-energy light particles are known to produce giant clusters with sizes exceeding $100 \mathrm{~nm}$. These clusters are supposed to be carriers of electrical conductivity in ion irradiated polymers and influence the optical properties of such materials ${ }^{(18)}$. Several experimental approaches exist to study the clusters. Among these are: small angle X-ray scattering (SAXS), transmission electron microscopy, scanning tunneling microscopy, scanning probe microscopy, magnetic force microscopy, atomic force microscopy and UV-visible spectrometry. UV-visible spectroscopy is sensitive to the optically active cluster region i.e., visible cluster size. The formation of these carbonaceous clusters can be studied from the absorption edge of ultravioletvisible (UV-VIS) spectra of irradiated polymers. UV-VIS spectroscopy gives an idea about the value of optical band gap $\left(\mathrm{E}_{g}\right)$ with Tauc's expression ${ }^{(19)}$.

In the present work we have chosen an important plastic CR-39, which it is made from allyl diglycol carbonate monomer appears to have the following properties; are colorless and completely transparent to the visible light and almost completely opaque in infrared and ultraviolet region of the spectrum. For these reasons, it is largely used for the production of sun glass lenses. They can be colored by surface dyeing or bulk tinting; being highly isotropic and homogenous; have high abrasion resistance and high-quality optical properties; keep their excellent optical properties despite long-term exposure to chemicals like solvents; highly oxidizing acids, and strong basis and resist to heat distortion up to $100{ }^{\circ} \mathrm{C}$.

This plastic is made by polymerization of the oxydi-2, 1-ethanediyl, di-2-propenyl ester of carbonic acid. The monomer is an allyl resin containing the following functional group:

$$
\left[\mathrm{CH}_{2}=\mathbf{C H}-\mathrm{CH}_{2}-\right]
$$

The monomer itself contains two of these functional groups and has the following structure: 


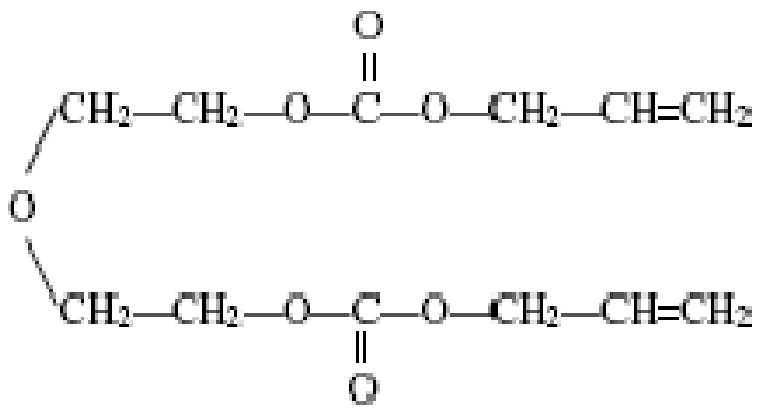

The branching point in this net is the tertiary carbon in the polyallyl chain ${ }^{(20)}$. Out of three links originating from this point two form the polyallyl chain and one consists of diethyleneglycol dicarbonate.

Because of the presence of the two allyl functional groups, the monomer can not only polymerize but also cross-link which results in a thermoset plastic characterized by being hard, infusible and insoluble in all solvents. In addition, CR39 homopolymer is a high-grade optical plastic whose index of refraction is just slightly less than that of crown glass.

The monomer is polymerized by adding a few percent of the catalyst di-isopropyl peroxy dicarbonate (IPP) to the monomer, and then heating. In general the chemical formula for CR-39 may be written as $\left(\mathrm{C}_{12} \mathrm{H}_{18} \mathrm{O}_{7}\right)_{n}$, where $\mathrm{H}$ content is $6.6 \% \mathrm{wt}, \mathrm{C}$ content is $52.6 \% \mathrm{wt}$ and $\mathrm{O}$ content is $40.8 \%$ wt.

Many other trials were made to study the effect of gamma radiations on the UV-VIS spectra of polymers (using chemical etching) however in a different context. Singh and Prasher ${ }^{(13)}$ have quoted decrease in band gap with increase in gamma dose without mentioning whether the band gap was direct or indirect. Saad et al. ${ }^{(21)}$ have indicated qualitatively the decrease in the band gap with increasing gamma dose with maximum up to $400 \mathrm{kGy}$. El-Shahawy ${ }^{(22)}$ has reported the slight decrease in direct band gap with increase in gamma dose up to a maximum of $100 \mathrm{kGy}$. Tanu Sharma et al. ${ }^{(23)}$ studied the effect of gamma dose (maximum up to $800 \mathrm{kGy}$ ) on the absorption and band gap of CR-39 polymer by using UV-VIS spectrophotometery.

In the present work, we report the effect of gamma radiations on the UV-VIS spectra of CR-39 plastic track detector as the spectroscopic studies. Also we investigated the behaviours of band gap and Urbach energy with increasing gamma dose. The simultaneous existence of indirect and direct band gap in CR-39 polymer and the number of carbon atoms $(\mathrm{N})$ in a cluster are also reported.

\section{EXPERIMENTAL PROCEDURE}

CR-39 samples of thickness $500 \mu \mathrm{m}$, type TASTRAK (Track Analysis System Ltd., UK) (composition $\mathrm{C}_{12} \mathrm{H}_{18} \mathrm{O}_{7}$, molecular weight 274 a.m.u., density $1.32 \mathrm{~g} / \mathrm{cm}^{3}$ ), were irradiated with different gamma doses using ${ }^{60} \mathrm{Co}$ source of 7.5 $\mathrm{kGy} / \mathrm{h}$ dose rate at Atomic Energy Authority, Cairo, Egypt.
The samples were irradiated for various times and the total gamma doses obtained were listed in table 1. The error in doses ranges from $8 \%$ for low dose to about $1 \%$ for high doses.

The nature of the optical modifications of the pristine and gamma irradiated CR-39 polymer samples were subjected to spectral studies in the Ultraviolet and Visible region. These studies were carried out by using TECHCOMP Double beam Double monochromator UV-Visible Spectrophotometer (UVVIS 8500) in the wavelength range of 190-1100 nm having resolution of $0.1 \mathrm{~nm}$. All the spectra were recorded by mounting the samples in the Integrating Sphere Assembly attached with the Spectrophotometer, keeping air as the reference.

\section{RESULTS AND DISCUSSION}

The optical absorption spectra of pristine and gamma irradiated CR-39 samples were recorded using UV-Visible spectrophotometer in the wavelength range, $190-1100 \mathrm{~nm}$ at room temperature. From these spectra, displayed in Fig. 1, it is clear that a shift of absorption edge towards longer wavelength with increasing gamma absorbed dose can be readily observed. The absorption peak with increasing dose is seen to change into a broad one. This behavior is generally interpreted as caused by the formation of extended systems of conjugate bonds i.e. possible formation of carbon clusters. The absorption bands in the investigated range of wavelength are associated to the $\pi-\pi^{*}$ electronic transitions. This type of transitions occurs in the unsaturated centers of the molecules i.e. in compounds containing double or triple bonds and also in aromatics. The excitation of $\pi$ electron requires smaller energy and hence, transition of this type occurs at longer wavelengths.

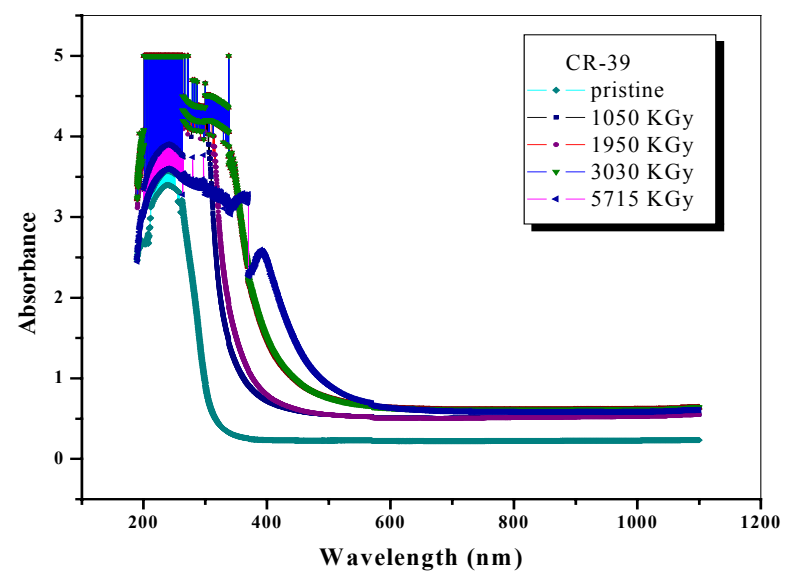

FIG. 1: UV-visible spectra of the pristine and gamma-irradiated CR39.

The optical absorption coefficient $(\alpha)$ was calculated from the absorbance, $(A)$. After correction for reflection losses, $\alpha$ may be obtained using the following equation ${ }^{(26)}$ :

$$
\alpha(v)=2.303 A / l
$$


TABLE I: The variation of band gap energy and Urbach's energy in the pristine and gamma irradiated CR-39, along with the number of carbon atoms $(\mathrm{N})$ per conjugated length.

\begin{tabular}{|l|l|l|l|l|l|}
\hline \multirow{2}{*}{ Gamma dose (kGy) } & \multicolumn{3}{|l|}{ Band gap energy (eV) } & \multirow{2}{*}{ Urbach's energy $(\mathbf{e V})$} & $\mathbf{N}$ \\
\cline { 5 - 6 } & Indirect & Direct & & Indirect & Direct \\
\hline pristine & 3.6 & 4.2 & 0.55 & $\sim 5$ & $\sim 4$ \\
1050 & 3.1 & 3.8 & 0.61 & $\sim 5$ & $\sim 5$ \\
1950 & 2.8 & 3.7 & 0.59 & $\sim 6$ & $\sim 5$ \\
3030 & 2.2 & 0.56 & $\sim 7$ & $\sim 6$ \\
5715 & 1.9 & 2.7 & 0.53 & $\sim 9$ & $\sim 7$ \\
\hline
\end{tabular}

where $l$ is the sample thickness in $\mathrm{cm}$ and $A$ is defined by $A$ $=\log \left(I_{O} / I\right)$ where $I_{O}$ and $I$ are the intensity of the incident and transmitted beams, respectively. The absorption coefficient near the band edge for non-crystalline materials shows an exponential dependence on the photon energy $(h v)$ which follows the Urbach formula ${ }^{(27)}$ :

$$
\alpha(v)=\alpha_{o} \exp \left(h v / E_{u}\right)
$$

where $\alpha_{o}$ is a constant, $E_{u}$ is an energy which is interpreted as the width of the tail of localized states in the forbidden band gap, $v$ is the frequency of radiation and $h$ is Planck's constant. The origin of $E_{u}$ is considered as thermal vibrations in the lattice $^{(28)}$. The logarithm of the absorption coefficient $\alpha(v)$ was plotted as a function of the photon energy $(h v)$ for CR39 irradiated with different doses of gamma as in Fig. 2. The values of the Urbach energy $\left(E_{u}\right)$ were calculated by taking the reciprocal of the slopes of the linear portion in the lower photon energy region of these curves and listed in Table 1. The decrease in the Urbach's energy in case of CR-39 may be due to the decrease in the crystalline nature of the polymer.

The above relation was first proposed by Urbach ${ }^{(27)}$ to describe the absorption edge in alkali halide crystals. Also, this relation has been found to hold for many amorphous materials. Eq. (2) has been modified to a more general form by Davis and $\operatorname{Mott}^{(29)}$.

$$
\alpha(h v)=B\left(h v-E_{g}\right)^{n} / h v
$$

where $h v$ is the energy of the incident photons, $E_{g}$ is the value of the optical energy gap between the valence band and the conduction band, and $n$ is the power, which characterizes the electronic transition, whether it is direct or indirect during the absorption process in the $\mathbf{K}$-space. Specially, $n$ is $1 / 2,3 / 2$, 2 and 3 for direct allowed, direct forbidden, indirect allowed and indirect forbidden transitions, respectively.

The factor $B$ depends on the transition probability and can be assumed to be constant within the optical frequency range. The usual method for the determination of the value of $E_{g}$ involves plotting $(\alpha h v)^{1 / n}$ against $(h v)$. Indirect transitions in many amorphous materials fit the case for $n=2$; for a direct transition a reasonable fit with $n=1 / 2$ is achieved. In the present study the most satisfactory results were obtained by plotting $(\alpha h v)^{1 / 2}$ and $(\alpha h v)^{2}$ as a function of photon energy $(h v)$ respectively, taking into account the linear portion of the fundamental absorption edge of the UV-Visible spectra

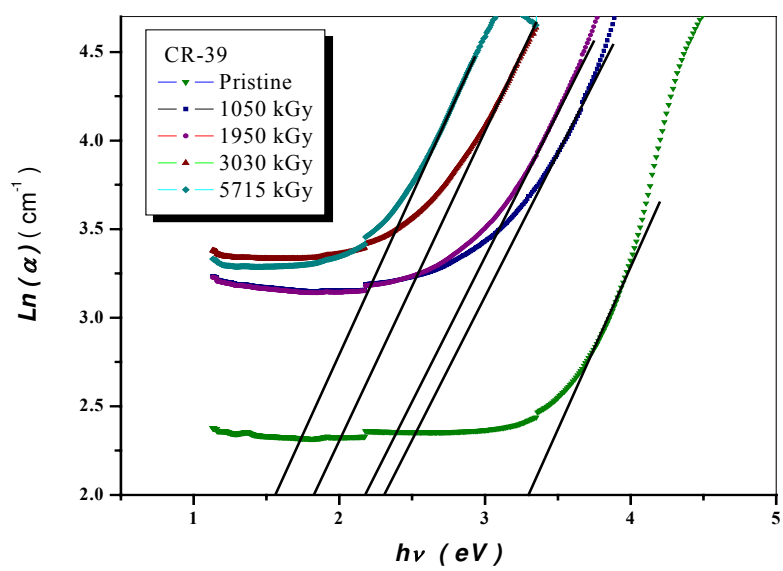

FIG. 2: The dependence of natural logarithm of $\alpha$ on photon energy for pristine and gamma irradiated CR-39 polymer.

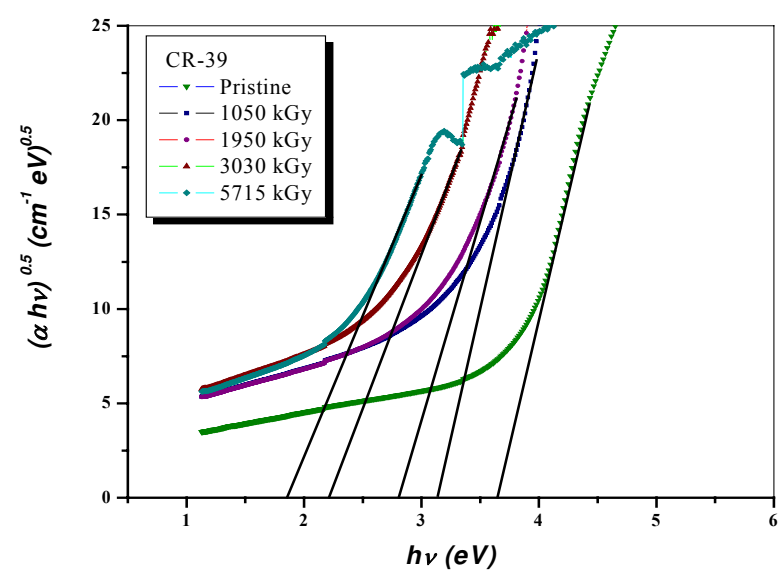

FIG. 3: The dependence of $(\alpha h v)^{1 / 2}$ on photon energy $(h v)$ for pristine and gamma irradiated CR-39 polymer.

(shown in Fig. 1). Such plots have been presented in Figs. 3 and 4 , respectively. From extrapolating the straight parts of these relations to the $h v$ axis, indirect and direct band gaps have been determined for pristine and gamma irradiated (different doses) CR-39 polymer and these different transition energies are displayed in Fig. 5 and depicted in Table 1. This, in turn, clearly indicates the simultaneous existence of indirect and direct band gap in CR-39 polymer with decreasing tendency at higher gamma dose. This result confirms that the 


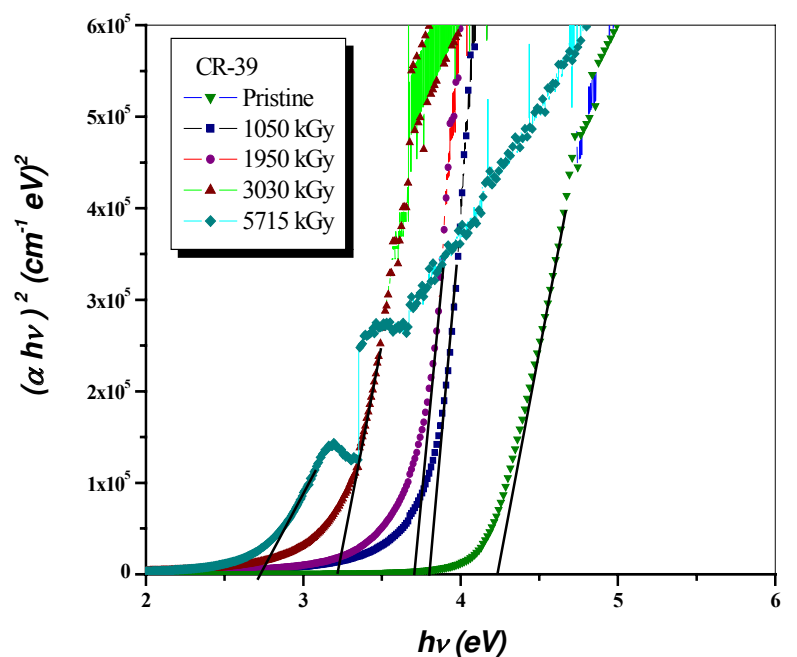

FIG. 4: The dependence of $(\alpha h v)^{2}$ on photon energy $(h v)$ for pristine and gamma irradiated CR-39 polymer.

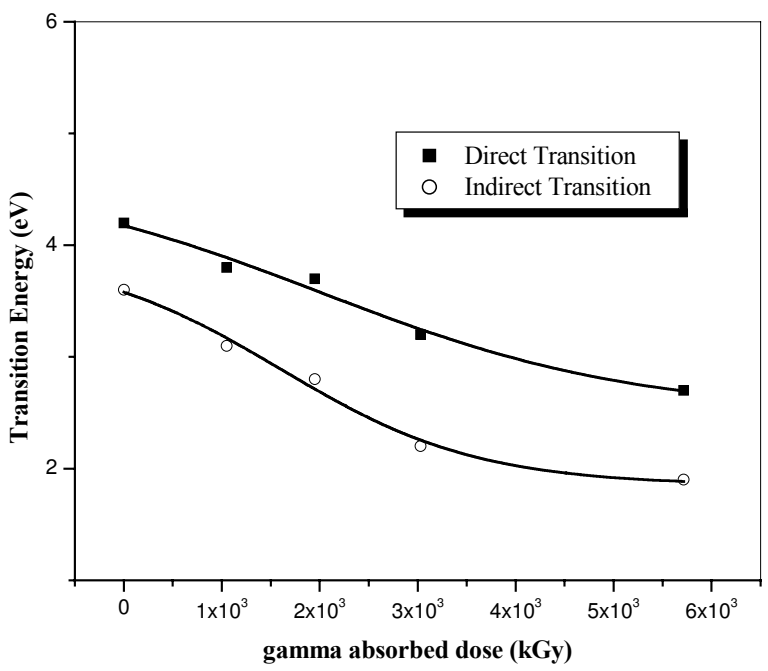

FIG. 5: Plots for transition energy (eV) as a function of gamma absorbed dose (kGy). irradiation produces faults in CR-39 polymer structure (band rupture, free radical, etc.) which increases the electronic disorder inducing the creation of a permit state in the forbidden (interdict) band or the deformation of the valence band.

Furthermore, the values of indirect band gap have been found to be lower than the corresponding values for the direct band gap as shown in Fig. 5. The simultaneous existence of indirect as well as direct band gap in CR-39 polymer has rarely been reported ${ }^{(23)}$, although such a coexistence of direct and indirect band gap have been observed in some other materials ${ }^{(24,25,30-34)}$.

Pristine CR-39 is transparent and has very little absorption. Upon irradiation, the colorless surface became light brown as the gamma dose gradually increased ${ }^{(22)}$. For a linear structure, the number of carbon atoms per conjugation length $N$ is given by ${ }^{(6)}$ :

$$
N=2 \beta \pi / E_{g}
$$

Here $2 \beta$ gives the band structure energy of a pair of adjacent $\pi$ sites. The value of $\beta$ is taken to be $-2.9 \mathrm{eV}$ as it is associated with $\pi \rightarrow \pi^{*}$ optical transitions in $-\mathrm{C}=\mathrm{C}-$ structure. As the shift of the absorption edge can be attributed to an increase of the conjugation length without formation of new lengthy linear conjugated structures, Eq. (4) is applied in the present study.

\section{CONCLUSIONS}

From this experimental study of pristine and gamma irradiated CR-39, the values of the optical band gap $\left(E_{g}\right)$, and Urbach energy $\left(E_{u}\right)$ were determined from the optical absorption spectra. It is concluded that the values of indirect band gap are lower than the corresponding values of direct band gap in pristine and gamma irradiated CR-39 polymer. And also the band gap $\left(\mathrm{E}_{g}\right)$ decreases with increase of gamma absorbed dose, due to the photo-degradation of CR-39 and the formation of defects and clusters in the material, while the cluster size (number of carbon atoms $(\mathrm{N})$ per conjugated length) increases with increase of absorbed dose for both cases.
[1] C. Darraud, B. Bennamane, J. L. Decossas, and J. C. Vareille, Nucl. Instr. and Meth. B 131, 198 (1997).

[2] A. Charlesby, Atomic Radiation and Polymers, Oxford Pergamon (1960).

[3] A. Chapiro, Radiation Chemistry of Polymeric Systems Interscience, New York 386 (1962).

[4] L. Calcagno, G. Compagnini, and G. Foti, Nucl. Instrum. Methods B 65, 413 (1992).

[5] J. C. Pivin, Nucl. Instrum. Methods B 84, 484 (1994).

[6] D. Fink, W. H. Chung, R. Klett, A. Schmoldt, J. Cardoso, R. Montiel, M. H. Vazquez, L. Wang, F. Hosoi, H. Omichi, and P. Goppelt-Langer, Radiat. Eff. Defects Solids 133, 193 (1995).

[7] A. Tidjani and Y. Watanabe, J. Polym. Sci. Part A: Polym.
Chem. 33, 1455 (1995).

[8] D. Sinha, G. K. Sarker, S. Ghosh, A. Kulshreshthta, K. K. Dwivedi, and D. Fink, Radiat. Meas. 29, 599 (1997).

[9] D. Sinha and K. K. Dwivedi, Radiat. Phys. Chem. 53, 99 (1998).

[10] T. Phukan, D. Kanjilal, T. D. Goswami, and H. L. Das, Nucl. Instrum. Methods; B 155, 116 (1999).

[11] R. Mishra, S. P. Tripathy, D. Sinda, K. K. Dwivedi, S. Ghosh, D. T. Khathing, M. Muller, D. Fink, and W. H. Chung, Nucl. Instrum. Methods B 168, 59 (2000).

[12] R. Mishra, S. P. Tripathy, K. K. Dwivedi, D. T. Khathing, S. Ghosh, M. Muller, and D. Fink, Radiat. Eff. Defects Solids 153, 257 (2001). 
[13] S. Singh and S. Prasher, Nucl. Instr. and Meth. B 222, 518 (2004).

[14] Basma A. El-Badry, M. F. Zaki, Tarek M. Hegazy, and A. Ahmed Morsy, Radiat. Eff. Defects Solids (2007) in press.

[15] D. Fink, R. Klett, L. T. Chadderton, J. Cardosa, R. Montiel, H. Vezquez, and A. Karanovich, Nucl. Instrum. Methods Phys. Res. B 111, 303 (1996).

[16] J. Davenas, P. Thevenard, G. Boiteux, M. Fallavier, and X. L. Lu, Nucl. Instrum. Methods Phys. Res. B 46, 317 (1990).

[17] T. Phukan, D. Kanijlal, T. Goswami, and H. Das. Radiat. Meas. 36, 611 (2003)

[18] D. Fink, M. Miller, L. T. Chadderton, P. H. Cannington, R. G. Elliman, and D. C. Mc Donald. Nucl. Instrum. Methods B 32 , 125 (1988)

[19] S. Gupta, D. Choudhary, and A. Sarma, J. Polym. Sci. Part B 38, 1589 (2000).

[20] J. Stejny, T. Portwood, Nucl. Tracks Radiat. Meas. 12, (1-6) 59 (1986).

[21] A. F. Saad, S. T. Awa, R. Yokota, M. Fuji Radiat Meas. 40, 780 (2005).

[22] M. A. El-Shahawy, Poly. Degr. and Stab. 57, 157 (1997).

[23] Tanu Sharma, Sanjeev Aggarwal, Shyam Kumar, V. K. Mittal, P. C. Kalsi , and V. K. Manchanda, J. Mater Sci. 42, 1127 (2007).
[24] A. M. Cruzman J. D. Carlson, J. E. Bares, and P. P. Pronko, Nucl. Instrum. Methods Phys. Res. B 7/8, 468 (1985).

[25] M. Mujahid, D. S. Srivastava, Shiuli Gupta, and D. K. Avasthi, Radiation Physics and Chemistry 74, 118 (2005).

[26] Mark Fox, Optical Properties of Solids, Oxford University Press Inc., New York (2001).

[27] F. Urbach, Phys. Rev. 92, 1324 (1953).

[28] M. D. Migahed, H. M. Zidan, Current Applied Physics 6, 91 (2006).

[29] N. F. Mott, E. A. Davies, Electronic Processes in NonCrystalline Materials, Clarendon Press, Oxford, (1979).

[30] C. J. Mathai, S. Saravanan, M. R. Anantharaman, S. Venkitachalam, and S. Jayalekshmi, J. Phys. D Appl. Phys. 35, 2206 (2002).

[31] A. B. M. Shah Jalal, S. Ahmed, A. H. Bhuiyan, and M. Ibrahim, Thin Solid Films 288, 108 (1996).

[32] G. Hirankumar, S. Selvasekarapandian, N. Kuwata, J. Kawamura, and T. Hattori, J. Power Sources 144, 262 (2005).

[33] Y. Yasaki, N. Sonoyama, and T. Sakata, J. Electroanalyt. Chem. 469, 116 (1999).

[34] Jin Moon-Seog, Kim Nam-Oh, Kim Hyung-Gon, Yoon ChangSun, Lee Choong-II, Kim Mi-Yang, and Kim Wha-Tek, J. Korean Phys. Soc. 4, 692 (2001). 